\title{
ХИРУРГИЧЕСКОЕ ЛЕЧЕНИЕ КРОВОТЕЧЕНИЙ ИЗ ВАРИКОЗНЫХ ВЕН ПИЩЕВОДА И ЖЕЛУДКА У БОЛЬНЫХ ДЕКОМПЕНСИРОВАННЫМ ЦИРРОЗОМ ПЕЧЕНИ
}

\author{
(С Котив Б.Н., Дзидзава И.И., Онницев И.Е., Хохлов А.В., Зубарев П.Н., \\ Солдатов С.А., Шевцов С.В., Парфенов А.О.
}

\section{Военно-медицинская академия им. С.М. Кирова, Санкт-Петербург}

E-mail: ionnicev@mail.ru

\begin{abstract}
В статье представлены результаты хирургического лечения кровотечений из варикозных вен пищевода и желудка у больных декомпенсированным циррозом печени. В исследование включены 55 пациентов. Средний возраст составил 50,5 12,7 года. Подавляющее большинство пациентов имели клинические признаки тяжелой печеночной недостаточности в соответствии с критериями Child-Turcotte-Pugh (класс В - 7, класс С - 48 больных). Среди больных было выделено две группы. В первой группе больным выполнено только эндоскопическое лигирование. Во второй применялось комбинированное лечение: лапароскопическая деваскуляризация желудка и пищевода с до- или интраоперационным эндоскопическим лигированием вен пищевода. Полученные результаты указали на повышение выживаемости пациентов во второй группе.
\end{abstract}

Ключевые слова: цирроз печени, портальная гипертензия, варикозное расширение вен пищевода и желудка, лапароскопическая деваскуляризация вен пищевода.

\section{SURGICAL TREATMENT OF BLEEDING FROM ESOPHAGEAL AND GASTRIC VARICES IN PATIENTS WITH DECOMPENSATED CIRRHOSIS}

Kotiv B.N., Dzidzava I.I., Onnicev I.E., Khokhlov A.V., Zubarev P.N., Soldatov A.S., Shevtsov S.V., Parfenov A.O. S.M. Kirov Military Medical Academy, St. Petersburg

The article presents the results of surgical treatment of bleeding from varicose veins of the esophagus and stomach in patients with decompensated liver cirrhosis. The study included 55 patients. The median age was $50.5 \pm 12.7$ years. The vast majority of patients had clinical signs of severe liver failure in accordance with the criteria Child-Turcotte-Pugh (class B -7 , class $\mathrm{C}-48$ patients). Two groups were distinguished among the patients. In the first group, patients underwent only endoscopic ligation. The second used a combination treatment: laparoscopic gastric and esophageal devascularization with pre-or intraoperative endoscopic ligation of esophageal veins. The obtained results indicated an increase in the survival rate of patients in the second group.

Keywords: liver cirrhosis, portal hypertension, varicose veins of the esophagus and stomach, laparoscopic devascularization of esophageal veins.

Кровотечение из варикозно расширенных вен пищевода (ВРВП) - грозное осложнение синдрома портальной гипертензии, являющееся одной из основных причин смерти у больных циррозом печени (ЦП) $[1,3,5]$. Летальность от первого кровотечения составляет $20-50 \%$, а при рецидиве геморрагии достигает 70\% [6, 12]. В 40\% наблюдений происходит рецидив кровотечения в последующие 6 недель наблюдения [5, 9]. Общая летальность при ЦП, осложненном кровотечениями из варикозно расширенных вен пищевода и желудка (ВРВПиЖ), колеблется от 39\% до 100\% [4, $5,16]$.

Лечение и профилактика пищеводножелудочных кровотечений портального генеза продолжает оставаться одной из трудных областей хирургической гепатологии. Для хирургической коррекции портальной гипертензии предложено более 200 способов оперативных вмешательств, что объясняется чрезвычайной сложностью проблемы, многообразием клинических проявлений синдрома и отсутствием абсолютно надежных методов хирургического лечения [3]. Сложность лечения больных данной категории обусловлена прежде всего тяжелой печеночной дисфункцией как лимитирующим фактором, определяющим результаты лечения [3, 17]. Ocoбую актуальность представляет проблема лечения осложнений портальной гипертензии у больных декомпенсированным ЦП, когда возможности оперативного лечения ограничены в силу низкого функционального резерва печени $[1,4,12]$.

Методом «золотого стандарта» лечения кровотечений из ВРВПиЖ является эндоскопическое лигирование (ЭЛ). Эффективность данного способа эндоскопического гемостаза при остром кровотечении составляет 86-95\%. Частота рецидивов в раннем послеоперационном периоде составляет $15-30 \%$. Летальность колеблется от $3,6 \%$ до 10,2\%. Рецидив варикозного расширения вен пищевода в отдаленном периоде диагностируется в 57,1-60\% случаев, а кровотечения развиваются у $10-20 \%$ больных. Прогрессирование варикозной трансформации вен желудка с риском развития 
кровотечения из них наблюдается у 18\% пациентов, что требует выполнения оперативного вмешательства [1, 2, 6, 12, 17].

Одним из наиболее эффективных средств предупреждения кровотечений из ВРВПиЖ является выполнение селективных и парциальных портокавальных анастомозов (ПКА). В ряде случаев выполнение ПКА возможно для лечения кровотечений после достижения временного гемостаза. Однако выполнение ПКА считается возможным у пациентов с компенсированной и субкомпенсированной функцией печени. Декомпрессивные венные анастомозы невозможны у больных с декомпенсированной функцией печени и при наличии тяжелых сопутствующих заболеваний. В данном случае рекомендуется рассматривать возможность выполнения трансюгулярного внутрипеченочного портосистемного шунта (TIPS) $[3,5,14,18]$.

При неэффективности эндоскопических методов обеспечения гемостаза и невозможности TIPS у больных с тяжелой печеночной недостаточностью возможно выполнение операции азигопортального разобщения (АПР): операции Таннеpa-Пациора, Sugiura-Futagawa и др. [7, 11, 13]. Преимущество данных операций заключается в сохранении обедненного портального притока к печени и разобщении анатомических связей портального бассейна с венами пищевода и желудка $[11,14,18]$. Частота рецидива пищеводножелудочного кровотечения после ЭЛ и азигопортального разобщения в отдаленном периоде сопоставима и составляет 20-21\% [9, 16].

В течение последних 10-15 лет в литературе появляется все большее число работ, оценивающих возможность и эффективность операции АПР лапароскопическим доступом. Впервые лапароскопическая деваскуляризация пищевода и желудка (ЛДПЖ) была выполнена в 1994 г. S. Kitano. B 2003 г. A. Helmy et al. выполнили лапароскопическую деваскуляризацию пищевода и желудка у 18 пациентов с хорошими результатами. Автор указывает на преимущество этих операций перед открытыми: они менее травматичны, переносятся больными легко, кровопотеря составляет 150-650 мл, что значительно снижает отрицательное влияние на функциональную активность печени. В 2004 г. J. Danis et al. сообщили о новом лапароскопическом вмешательстве (процедура Danis) [9]. Она состояла в выполнении деваскуляризации желудка и пищевода с использованием сосудистого коагулятора LigaSure и линейного сшивающего аппарата Endo-GIA для пересечения коротких вен желудка, ветвей коронарной вены желудка.

M. Tomikawa et al. в 2000 г. сообщили о хороших результатах после выполнения ЛДПЖ со спленэктомией у 10 больных с ВРВП, резистент- ными к ЭЛ, и гиперспленизмом [16]. У всех пациентов получены хорошие результаты: отсутствие рецидивов кровотечения, высокое качество жизни без энцефалопатии. Азигопортальное разъединение по Sugiura-Futagawa, выполняемое лапароскопическим доступом, стало популярным. Послеоперационная летальность в группе больных С по Child-Pugh не превышает 5\% [7, 10, 14].

Цель исследования: оценить результаты хирургического лечения и профилактики пищеводно-желудочных кровотечений у больных декомпенсированным циррозом печени.

\section{МАТЕРИАЛЫ И МЕТОДЫ ИССЛЕДОВАНИЯ}

Проведен анализ результатов лечения 55 больных циррозом печени. Мужчин было $30(54,5 \%)$, женщин 25 (45,5\%). Средний возраст составил 50,5 $\pm 12,7$ года. Подавляющее большинство пациентов имели клинические признаки тяжелой печеночной недостаточности в соответствии с критериями Child-Turcotte-Pugh (класс B -7 , класс C -48 больных). С продолжающимся пищеводно-желудочным кровотечением (ПЖК) поступило 20 (36,4\%) больных. В 35 (63,6\%) случаях на момент госпитализации в стационар имело место состоявшееся гастроэзофагеальное кровотечение. Для купирования кровотечения и обеспечения устойчивого гемостаза выполнялось ЭЛ. При его неэффективности формировались показания к лапароскопической деваскуляризации пищевода и желудка.

Эндоскопическое лигирование выполняли в ходе эзофагоскопии при помощи волоконного эндоскопа GIF Q 10 фирмы “Olympus” (Япония) с помощью набора для эндоскопического лигирования фирмы "Wilson-Cook".

Из 20 пациентов с продолжающимся ПЖК у 16 устойчивый гемостаз удалось достигнуть эндоскопическим лигированием. Источником кровотечения, как правило, были вены пищевода. Первую лигатуру накладывали либо непосредственно на место разрыва стенки вены, либо лигировали поврежденный венозный ствол ниже и выше уровня дефекта. Последующие лигатурные кольца накладывали на соседние варикозные узлы, размещая их выше и ниже в шахматном порядке. Особенность эндоскопического лигирования при кровотечении из варикозно расширенных вен кардиального отдела и свода желудка заключалась в том, что выявление кровотечения и лигирование кровоточащей вены выполняется при ретрофлексии гастроскопа. Вследствие обширной площади распространения ВРВЖ, сетчатого вида архитектоники этих сосудов, полностью произвести ЭЛ всех варикозных вен желудка не представлялось возможным. Поэтому для остановки 
кровотечения из ВРВЖ и предотвращения рецидивов кровотечений было крайне важно прицельно лигировать участок с дефектом венозной стенки. После остановки кровотечения из ВРВЖ устанавливался желудочный зонд с целью промывания желудка, его опорожнения и контроля гемостаза.

У 4 больных применить ЭЛ в качестве экстренного вмешательства для остановки кровотечения не представлялось возможным, так как обильное поступление крови в просвет пищевода не позволяло локализовать источник геморрагии. В этих наблюдениях ограничивались диагностической фиброэзофагогастроскопией с последующей постановкой зонда Блэкмора. Эндоскопическое лигирование в таких случаях выполняли в срочном порядке через 6-12 часов после достижения временного гемостаза.

В 35 (62,3\%) наблюдениях при эндоскопической картине состоявшегося кровотечения выполнено ЭЛ варикозно расширенных вен пищевода и желудка с целью профилактики рецидива.

Операция лапароскопической деваскуляризации пищевода и желудка (ЛДПиЖ) симультанно с эндоскопическим лигированием ВРВП выполнена для лечения 20 пациентов. В 15 случаях операция дополнена лигированием селезеночной артерии в связи с наличием у пациентов тромбоцитопении средней и тяжелой степени.

Показанием к ЛДПиЖ в комбинации с ЭЛ ВРВП являлась профилактика рецидива пищеводных кровотечений при неэффективности двух сеансов ЭЛ. У четырех пациентов показанием к ЛДПиЖ явился ранний рецидив геморрагии из ВРВЖ после ЭЛ. Для временной остановки кровотечения во всех случаях применялся зондобтуратор.

Согласно консенсуса Baveno V (2010) и «Клиническим рекомендациям по лечению кровотечений из варикозно расширенных вен пищевода и желудка» (2013) тактическими принципами лечения пациентов с варикозным пищеводножелудочным кровотечением в клинике являлись:

- Одномоментное начало медикаментозных мероприятий, включающих: назначение вазоконстрикторов (терлипрессина в/в дозе 2 мг, затем по 1 мг каждые 6 часов, не превышая суточную дозу более 120 мкг/кг веса тела); аналогов соматостатина (октреотид в дозировке 20 мг 4 раза в сутки); ингибиторов протонной помпы (омепразола) по 80 мг, а затем по 40 мг 2 раза в сутки в/в, с гемостатической целью назначение транексамовой кислоты (транексам) по 500-750 мг в/в капельно 2-4 раза в сутки;

- Компонентом гемостатической терапии было введение 300-600 мл одногруппной свежезамороженной плазмы; гемотрансфузию проводили больным при снижении уровня гемоглобина ниже 80 г/л и снижении гематокритного числа менее $24 \%$.

- Пациентам назначали гепатопротекторы и антибиотики (гептрал, гепа-мерц и цефбактам 2 г).

У всех пациентов проводился мониторинг центрального венозного давления (ЦВД). Инфузионная терапия для устранения дефицита объема циркулирующей крови (ОЦК) за счет коллоидных среднемолекулярных растворов, улучшающих реологические свойства крови, в сочетании с солевыми и глюкозированными растворами воздействовала на транскапиллярный обмен и миграцию в ткани активных компонентов коллоидных сред. Эффективность восполнения ОЦК оценивали по динамике ЦВД (целевые значения 8-12 мм рт. ст.) и почасовому диурезу (не менее 60 мл/час).

Для сравнения эффективности лечения проводили статистическую обработку полученных данных при помощи таблиц сопряженности 2 х 2 с помощью критерия хи-квадрат с поправкой Йетса на непрерывность.

\section{РЕЗУЛЬТАТЫ ИССЛЕДОВАНИЯ И ИХ ОБСУЖДЕНИЕ}

Для оценки результатов лечения кровотечений из ВРВПиЖ все пациенты разделены на 2 группы. В первой группе больным выполнено только эндоскопическое лигирование $(n=35)$. Во второй применялось комбинированное лечение: лапароскопическая деваскуляризация желудка и пищевода с до- или интраоперационным ЭЛ вен пищевода $(\mathrm{n}=20)$.

Пациенты групп сравнения сопоставимы по степени печеночной недостаточности. В обеих группах при фиброгастроскопии определялось BРВП III-IV степени по К.-J. Рaquet. По степени ВРВПиЖ группы были сопоставимы (Таблица 1).

Больные с продолжающимся кровотечением из ВРВП ( $\mathrm{n}=55)$
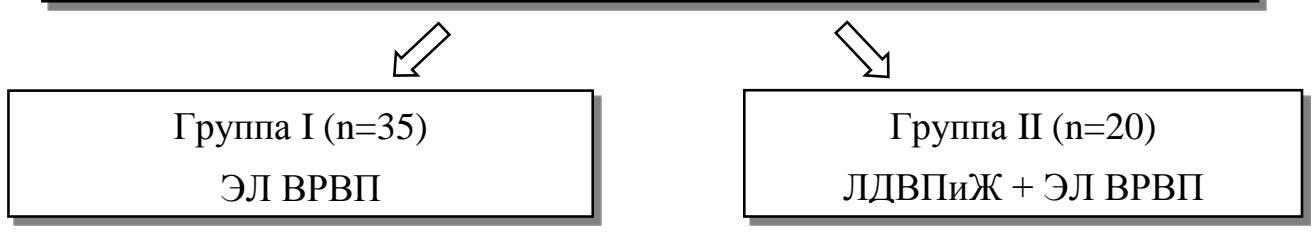

Рис. 1. Алгоритм формирования групп. 
Распределение больных по степени ВРВП по К.-J. Paquet

\begin{tabular}{|l|c|c|}
\hline \multicolumn{1}{|c|}{ Число больных } & III степень & IV степень \\
\hline Первая группа $(\mathrm{n}=35)$ & $24(68,5 \%)$ & $11(31,5 \%)$ \\
\hline Вторая группа $(\mathrm{n}=20)$ & $12(60 \%)$ & $8(40 \%)$ \\
\hline
\end{tabular}

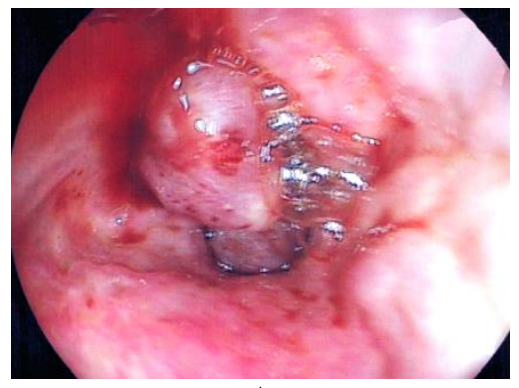

A

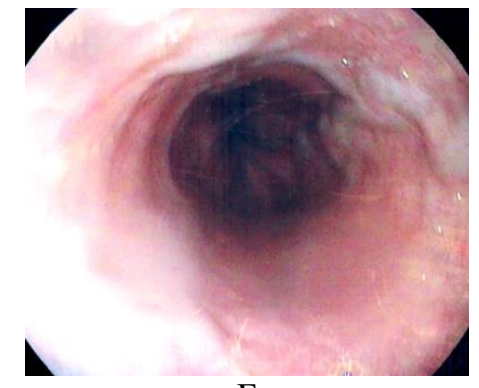

Б

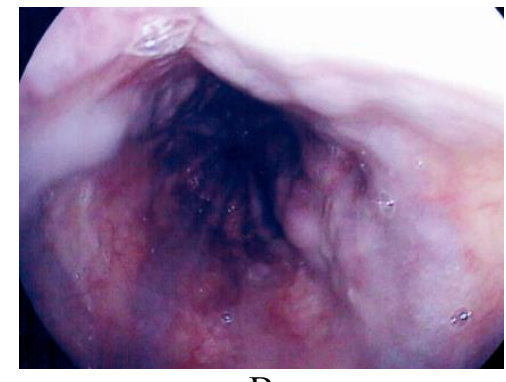

B

Рис. 2. Эндоскопическая фотография. Пациент Б.

А - ЭЛ варикозно расширенных вен пищевода латексными кольцами. Б - ВРВП ІІ ст. (через 1 месяц после ЭЛ). В - ВРВП ІІІ ст. (через 3 месяца после ЭЛ).

Оценивали эффективность гемостаза, а также частоту рецидива пищеводно-желудочных кровотечений в раннем и отдаленном послеоперационном периодах. Контрольную ФГДС осуществляли через 10-14 дней после купирования кровотечения и в отдаленном периоде наблюдения (через 1 месяц и далее 1 раз в 3 месяца в течение года).

Эффективность лигирования варикозно расширенных вен пищевода в лечении острого кровотечения составила 92,3\%. В четырех наблюдениях кровотечение возобновилось спустя 2 и 4 часа от момента ЭЛ. В данных случаях временный гемостаз достигнут при помощи зонда Блэкмора. После стабилизации состояния пациенты были оперированы. Выполнена ЛДЖП с интраоперационным ЭЛ. При кровотечении из вен кардиального отдела желудка эффективность ЭЛ достигала только $57,1 \%$.

В раннем послеоперационном периоде после ЭЛ пациентам с сотоявшимся кровотечением рецидивы геморрагий развились у 5 (14,3\%) больных. В данных случаях причиной рецидива пищеводных геморрагий явилось раннее отторжение лигатуры. Окончательный гемостаз достигнут повторным эндоскопическим лигированием.

Летальность в раннем послеоперационном периоде составила $11,4 \%(\mathrm{n}=4)$, все пациенты из группы поступивших с продолжающимся кровотечением. В трех случаях причиной смерти было прогрессирование печеночно-клеточной недостаточности и декомпенсация сопутствующей патологии. Развитие рецидива кровотечения из ВРВП явилось причиной летального исхода у одного больного.

При обследовании в отдаленном периоде (от 3 до 6 месяцев) у $19(65,5 \%)$ пациентов наблюдался рецидив ВРВП, а у $6(20,6 \%)$ больных диагности- ровано развитие варикозной трансформации вен свода желудка.

В отдаленном периоде наблюдения рецидивы пищеводно-желудочных кровотечений развились у девяти $(25,7 \%)$ больных циррозом печени. В двух наблюдениях источником кровотечений были эрозии желудка на фоне прогрессирования портальной гипертензивной гастропатии. У семи пациентов причиной кровотечения являлись варикозно расширенные вены пищевода и желудка. Рецидив геморрагий развивался у семи пациентов с однократным сеансом ЭЛ и у двух пациентов после двукратной процедуры. Большая часть пищеводных кровотечений развивалась через 3 и 6 месяца после предыдущего эндоскопического вмешательства и купированы повторным сеансом ЭЛ. Гастроэзофагеальное кровотечение явилось причиной летального исхода в периоде (3-6 месяцев) у 5 (41,6\%) больных I группы. В 58,4\% случаев причиной смерти больных I группы в отдаленные сроки было прогрессирование печеночной недостаточности (таблица 2).

Во второй группе больных полноценная деваскуляризация венозной системы желудка и пищевода приводила к регрессу ВРВПиЖ с III-IV до III степени. В раннем послеоперационном периоде отмечено 3 рецидива кровотечения. У одного больного на 10-е сутки после операции рецидив кровотечения из ВРВП остановлен эндоскопическим лигированием. В двух случаях причиной геморрагии явилось тотальное эрозивное поражение желудка с диффузной кровоточивостью. Летальный исход в раннем послеоперационном периоде имел место в одном случае $(5 \%)$. Причина смерти - прогрессирующая печеночно-клеточная недостаточность. 
Таблица 2

Частота рецидивов пищеводного кровотечения, рецидив ВРВП и летальность у больных I и II группы

\begin{tabular}{|c|c|c|c|c|c|c|c|c|}
\hline $\begin{array}{c}\text { Сроки } \\
\text { наблюдения, } \\
\text { мес. }\end{array}$ & \multicolumn{2}{|c|}{$\begin{array}{c}\text { Число больных, } \\
\mathrm{n}\end{array}$} & \multicolumn{2}{c|}{$\begin{array}{c}\text { Рецидивы } \\
\text { кровотечения, } \\
\mathrm{n} \mathrm{( \% )}\end{array}$} & \multicolumn{2}{c|}{$\begin{array}{c}\text { Рецидив ВРВП } \\
\text { (высокий риск } \\
\text { кровотечения), }(\%)\end{array}$} & \multicolumn{2}{c|}{$\begin{array}{c}\text { Летальность } \\
\text { общая / летальность } \\
\text { от кровотечения, } \mathrm{n}\end{array}$} \\
\cline { 2 - 9 } & $\mathrm{I}$ гр. & II гр. & I гр. & II гр. & I гр. & II гр. & I гр. & II гр. \\
\hline 1 & 35 & 20 & $7(20)$ & $3(16,7)$ & - & - & $4 / 1$ & $1 / 1$ \\
\hline 3 & 31 & 19 & $4(12,9)$ & $2(11,1)$ & $19(61,2)$ & $9(50)$ & $5 / 2$ & $1 / 1$ \\
\hline 6 & 26 & 18 & $5(19,2)$ & $2(11,7)$ & $14(53,8)$ & $5(29,4)$ & $7 / 2$ & $2 /-$ \\
\hline 12 & 19 & 16 & $5(26,3)$ & $2(13,3)$ & $11(57,8)$ & $4(26,6)$ & $4 / 2$ & $2 / 1$ \\
\hline 24 & 15 & 14 & $3(20)$ & $2(15,3)$ & $6(40)$ & $7(53,8)$ & $3 / 1$ & $3 /-$ \\
\hline 36 & 12 & 11 & $4(33,3)$ & $3(30)$ & $6(50)$ & $4(40)$ & $4 / 2$ & $4 / 1$ \\
\hline
\end{tabular}

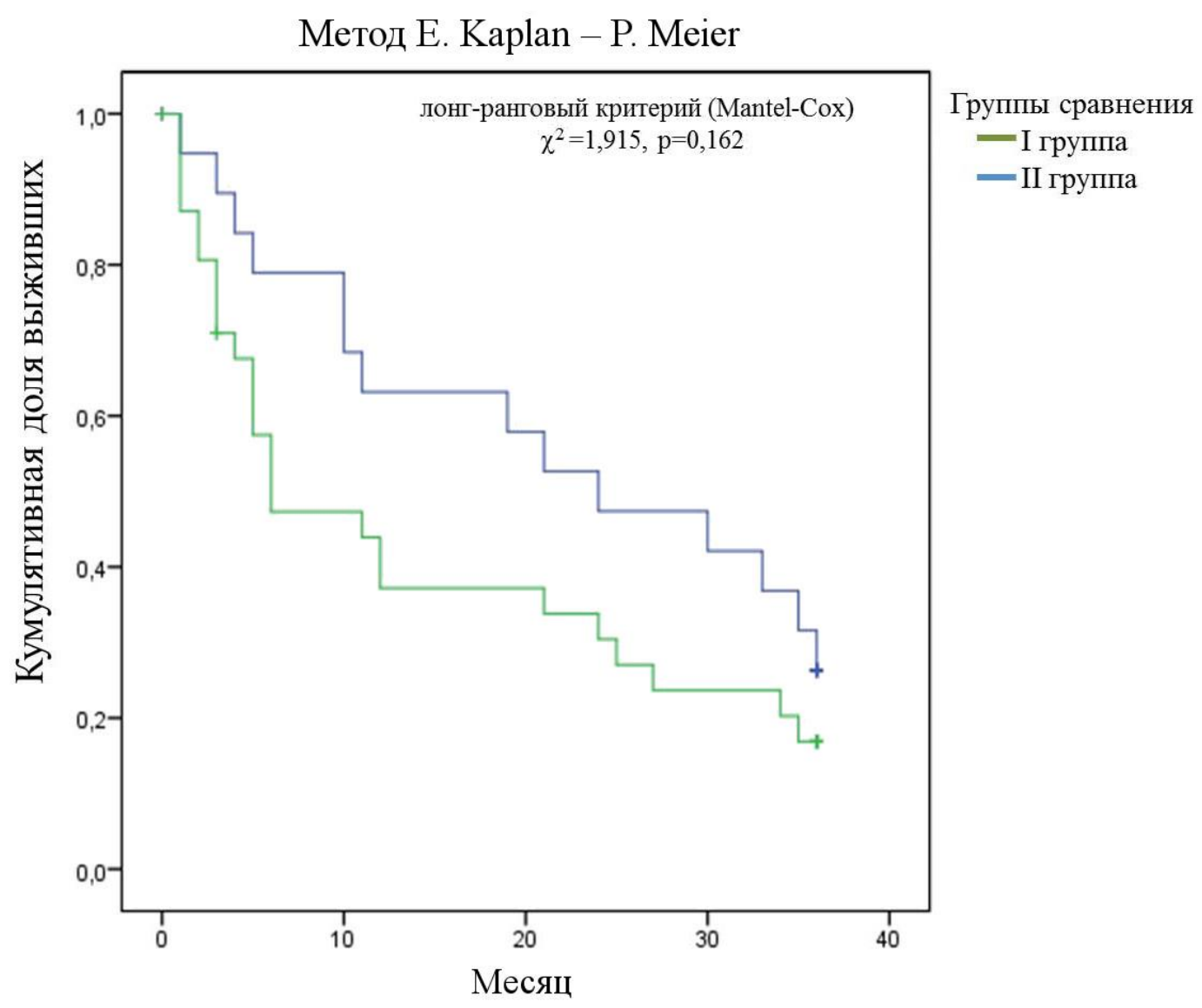

Рис. 3. Динамика выживаемости пациентов в группах сравнения (нижняя линия - I группа сравнения, верхняя - II группа).

Частота рецидивов кровотечения в раннем послеоперационном периоде во второй группе $15 \%$.Частота портальной гастропатии через 6 месяцев после операции снизилась с $65,8 \%$ до $15,8 \%$, через год - до $3,7 \%$.

У четырех пациентов отмечены рецидивы кровотечений через 3 и 6 месяцев после операции. Во всех случаях кровотечение было успешно остановлено ЭЛ.

Для сравнения эффективности лечения в двух группах больных провели статистическую обработку полученных данных при помощи таблиц сопряженности $2 \times 2$ с помощью критерия хиквадрат с поправкой Йетса на непрерывность.
Операция ЛДЖП с ЭЛ снижает летальность от пищеводно-желудочного кровотечения, по сравнению с проведением только ЭЛ, в течение шести месяцев после операции на $21,8 \%\left(\chi^{2}=2,61\right.$; $\mathrm{p}=0,106) ;$ в течение года на $25,5 \% \quad\left(\chi^{2}=2,75\right.$; $\mathrm{p}=0,091)$; в течение двух лет после операции на $25,4 \%\left(\chi^{2}=1,47 ; \mathrm{p}=0,225\right)$; в течение трех лет на $25,5 \%\left(\chi^{2}=0,43 ; \mathrm{p}=0,051\right)$.

При этом важно отметить, что в течение года после проведения ЛДЖП с ЭЛ снижается летальность от развития печеночной недостаточности на $18,1 \%\left(\chi^{2}=2,05 ; p=0,152\right)$ по сравнению с I первой группой, где ЭЛ являлся самостоятельным методом лечения. 
Частота пищеводно-желудочного кровотечения и рецидив ВРВПиЖ по эндоскопическим признакам в период наблюдения до 6 месяцев в обеих группах были сопоставимы. Через полгода после операции риск гастроэзофагеальных геморрагий портального генеза существенно ниже во второй группе больных, которым была выполнена лапароскопическая деваскуляризация с ЭЛ. Важно отметить, что ЛДЖП с ЭЛ снижает риск развития рецидива ВРВП в первый год после операции на $20 \%\left(\chi^{2}=2,61 ; \mathrm{p}=0,106\right)$ по сравнению с проведением только эндоскопического лигирования. Через 2 и 3 года результаты свидетельствуют о преимуществах сочетания лапароскопической деваскуляризации желудка и пищевода с ЭЛ по сравнению с эндоскопическим лигированием как изолированного метода лечения.

Рецидив пищеводно-желудочного кровотечения портального генеза в сроки 3-6 месяцев после операции ЛДВПиЖ явился причиной гибели больных в одном случае.

Выживаемость пациентов в группе II оказалась выше аналогичных показателей в группе I на всех этапах наблюдения, хотя статистически достоверных отличий между группами не отмечено $(\mathrm{p}=0,162)$ (рисунок 3$)$.

Таким образом, больные циррозом печени с декомпенсированной печеночной недостаточностью удовлетворительно переносят эндовидеохирургические вмешательства. Лапароскопическая деваскуляризация желудка с пересечением основных притоков к варикозным венам пищевода является малотравматичным и эффективным методом лечения пищеводножелудочных кровотечений при неэффективности ЭЛ и невозможности выполнения ПКА.

\section{ЛИТЕРАТУРА / REFERENCES}

1. Абдураханов Б.А. Лечение кровотечений из варикозно расширенных вен пищевода и желудка при циррозе печени // Анналы хирургической гепатологии. - 2006. - Т. 11, № 3. - С. 157. [Abdurakhanov B.A. Treatment of bleeding from varicose veins of the esophagus and stomach with cirrhosis. Annaly khirurgicheskoy gepatologii. 2006; 11(3): 157 (in Russ.)].

2. Габриэль С.А., Дурлештер В.М., Гучетль А.Я., Дынько В.Ю., Мурашко Д.С., Крушельницкий В.С. Эффективность эндоскопического лигирования варикозно расширенных вен пищевода // Кубанский научный медицинский вестник. 2016. - № 5(160). - C. 19-25. [Gabriel S.A., Durleshter V.M., Guchetl A.Y., Dynko V.Y., Murashko D.S., Krushelnitsky V.S. Efficacy of esophageal varices endoscopic ligation. Kubanskiy nauchnyy meditsinskiy vestnik. 2016; 160(5): 19-25 (in Russ.)].

3. Назыров Ф.Г., Девятов А.В., Бабаджанов А.Х., Джуманиязов Д.А., Байбеков Р.Р. Эффективность эндоскопических вмешательств у больных циррозом печени // Анналы хирургической гепатологии. - 2017. - Т. 22, № 2. - С. 45-53. [Nazyrov F.G., Devyatov A.V., Babadzhanov A.Kh., Dzhumaniyazov D.A., Baybekov R.R. The Effectiveness of Endoscopic Interventions in Liver Cirrhosis Patients. Annaly khirurgicheskoy gepatologii. 2017; 22(2): $45-53$ (in Russ.)].

4. Зубарев П.Н. Развитие идеи хирургического лечения больных портальной гипертензией в Военно-медицинской академии. - СПб. : Изд-во ВМедА, 2000. - 24 с. [Zubarev P.N. Development of the idea of surgical treatment of patients with portal hypertension in the Military Medical Academy. SPb. : Izd-vo VMedA; 2000: 24 (in Russ.)].

5. Котив Б.Н., Дзидзава И.И., Солдатов С.А., Кашкин Д.П., Алентьев С.А., Смородский А.В., Слободяник А.В., Онинцеев И.Е. Результаты селективного и парциального портокавального шунтирования и прогностические факторы долгосрочной выживаемости больных циррозом печени // Анналы хирургической гепатологии. 2015. - T. 20, № 2. - C. 46-58. [Kotiv B.N., Dzidzava I.I., Soldatov S.A., Kashkin D.P., Alentiev S.A., Smorodskiy A.V., Slobodyanik A.V., Onincev I.E. Results of the Selective and Partial Portacaval Bypass and Prognostic Factors of Longterm Survival in Patients with Liver Cirrhosis. Annaly khirurgicheskoy gepatologii. 2015; 20(2): 46-58 (in Russ.)].

6. Шерцингер А.Г., Жигалова С.Б., Семенова Т.С., Мартиросян Р.A. Роль эндоскопии в выборе лечения больных портальной гипертензией // Анналы хирургической гепатологии. - 2015. T. 20, № 2. - C. 20-30. [Shertsinger A.G., Zhigalova S.B., Semenova T.S., Martirosyan R.A. Role of Endoscopy in the Treatment of Portal Hypertension Patients. Annaly khirurgicheskoy gepatologii. 2015; 20(2): 20-30 (in Russ.)].

7. Akahoshi T., Uehara H., Tomikawa M., Kawanaka H., Hashizume M., Maehara Y. Comparison of open, laparoscopic, and hand-assisted laparoscopic devascularization of the upper stomach and splenectomy for treatment of esophageal and gastric varices: a single-center experience // Asian J Endosc Surg. - 2014. - Vol. 7, N 2. - P. 138-144. - DOI: 10.1111/ases.12096.

8. Costa Lacet C.M., Neto J.B., Ribeiro L.T., Oliveira F.S., Wyszomirska R.F., Strauss E. Schistosomal portal hypertension: Randomized trial comparing endoscopic therapy alone or preceded by esophagogastric devascularization and splenectomy // Ann Hepatol. - 2016. - Vol. 15, N 5. - P. 738-744. DOI: $10.5604 / 16652681.1212528$.

9. Danis J., Hubmann R., Pichler P., Shamiyeh A., Wayand W.U. Novel technique of laparoscopic azygoportal disconnection for treatment of esophageal varicosis: preliminary experience with five patients // Surg Endosc. - 2004. - Vol. 18, N 4. - P.702-705. DOI: $10.1007 / \mathrm{s} 00464-003-9096-7$.

10. Hashizume M., Tanoue K., Morita M., Ohta M., Tomikawa M., Sugimachi K. Laparoscopic gastric devascularization and splenectomy for sclerotherapy- 
resistant esophagogastric varices with hypersplenism // J Am Coll Surg. - 1998. - Vol. 187, N 3 - P. 263270.

11. Helmy A., Abdelkader Salama I., Schwaitzberg S.D. Laparoscopic esophagogastric devascularization in bleeding varices // Surg Endosc. - 2003. - Vol. 17, N 10. - P. 1614-1619. - DOI: 10.1007/s00464-0028928-1.

12. Jiang M., Liu F., Xiong W.J., Zhong L., Xu W., Xu F., Liu Y.B. Combined MELD and blood lipid level in evaluating the prognosis of decompensated cirrhosis // World J Gastroenterol. - 2010. - Vol. 16, N 11. P. 1397-1401.

13. Kitano S., Tomikawa M., Iso Y., Hashizume M., Moriyama M., Sugimachi K. Laparoscopy-assisted devascularization of the lower esophagus and upper stomach in the management of gastric varices // Endoscopy. - 1994. - Vol. 26, N 5 - P. 486-487. DOI: $10.1055 / \mathrm{s}-2007-1009006$.

14. Kobayashi T., Miura K., Ishikawa H., Soma D., Zhang Z., Ando T., Yuza K., Hirose Y., Katada T., Takizawa K., Nagahashi M., Sakata J., Kameyama H., Wakai T. Hand-assisted laparoscopic Hassab's procedure for esophagogastric varices with portal hypertension // Surg Case Rep. - 2017. - Vol. 3, N 1. - P. 111. - DOI: 10.1186/s40792-017-0387-y.

15. Salama I.A., Helmy A., Connolly R., Schwaitzberg S.D. Laparoscopic devascularization of the lower esophagus and upper stomach: experimental study in the pig // J Laparoendosc Adv Surg Tech A. - 2003. Vol. 13, N 1. - P. 59-63. - DOI: $10.1089 / 109264203321235502$.

16. Tomikawa M., Hashizume M., Saku M., Tanoue K., Ohta M., Sugimachi K. Effectiveness of gastric devascularization and splenectomy for patients with gastric varices // J Am Coll. Surg. - 2000. - Vol. 191, N 5. - P. 498-500.

17. Yoshida H., Mamada Y., Taniai N., Tajiri T. New methods for the management of esophageal varices // World J Gastroenterol - 2007. - Vol. 13, N 11. P. 1641-1645.

18. Zilberstein B., Sallet J.A., Ramos A., Eshkenazy R. Video laparoscopy for the treatment of bleeding esophageal varices // Surg Laparosc Endosc. - 1997. Vol. 7, N 3. - P. 185-191. 